\title{
Impact of Psychological Rehabilitation on Healing of Burn Patients
}

\author{
DALIA M. MEFREH ELSAKA, M.D.*; AYMAN A. ELHDAD, M.D.**; AHMED S. ELGAMAL, M.D.*; \\ MEDHAT S. HASAN, M.D.* and MOHAMED MAGDY MOHAMED MOWAFY, M.Sc.*
}

The Departments of General Surgery* and Psychiatry**, Faculty of Medicine, Menofia University

\begin{abstract}
Background: Psycological distress among people who have sustained burns is common. The time taken for burn wounds to heal can not be fully explained by physical factors such as burn type, wound size and depth only. Clinicians should not ignore the importance of psycological influences on the wound healing process and also give consideration to the potential for psycological rehabilitation to lessen patient's distress and improve wound healing outcomes.
\end{abstract}

Aim of Study: The aim is assessment of psychiatric status of burn patients during hospitalization, determination of psycological intervention methods and observation of impact of psycological rehabilitation on healing of burn patients.

Patients and Methods: The present non-randomized, prospective, cohort study was conducted on 40 patients with partial or full-thickness burns that attended Burn Unit of Plastic Surgery Department, Menoufia University Hospital between January and December 2019. Approval of the ethical committee and written informed consent from all participants was obtained.

Results: In the present study, in the pre-intervention period, we found that $35 \%$ of the patients had mild-to-moderate anxiety and most of the patients had scaled burn (55\%) and the mean burn surface area was $18.45 \pm 8.51 \%$. The majority of the patients had mixed burn degree $(57.5 \%)$.

Conclusion: Psychological interventions appear to effectively reduce burn patient psychological morbidities. We demonstrated that psychological interventions significantly reduce the risk of depression, anxiety, and hospital stay compared to usual care. These findings are very important as it confirms the promising role of psychological interventions in burn patients. Nevertheless, further studies with rigorous design, large sample size and multiregional cooperation are required.

Key Words: Acute stress disorder - Burn model system - Posttraumatic stress disorder - Total body surface area -Beck depression inventory-II.

Correspondence to: Dr. Dalia M. Mefreh Elsaka, The Department of General Surgery, Faculty of Medicine, Menofia University

\section{Introduction}

BURN is a traumatic injury to the skin or other organic tissue due to energy transfer most commonly in the form of heat (thermal burn). This may be a consequence of exposure to flames or scalding liquids through direct contact, inhalation or radiation. There are other special types of burn like electrical and chemical burn [1].

Few areas of medicine are as challenging medically and surgically as burn care. Burn injuries affect the very young and the very old, both men and women. Burn injuries can vary from small wounds that can be easily managed in the outpatient clinic to extensive injuries resulting in multiorgan system failure, a prolonged hospital stay and long term functional and psychosocial sequelae [2]

Psycological distress among people who have sustained burns is common. The time taken for burn wounds to heal can not be fully explained by physical factors such as burn type, wound size and depth only. Clinicians should not ignore the importance of psycological influences on the wound healing process, and also give consideration to the potential for psycological rehabilitation to lessen patients' distress and improve wound healing outcomes [3].

There are fewer psychological studies specific to burns, however those studies that have been conducted show similar patterns. For example, it was found that patients who engaged in positive psychological coping strategies, such as progressive muscular relaxation and deep-breathing during painful dressing changes, were found to leave hospital on average 1 week earlier than the control group [4]. 
With the increased survival of patients with large burns comes a new focus on the psychological challenges and recovery that such patients must face. Most burn centres employ social workers, vocational counsellors, and psychologists as part of the multidisciplinary burn team. Physiological recovery of burn patients is seen as a continual process divided into three stages (resuscitative or critical, acute, and long term rehabilitation). The psychological needs of burn patients differ at each stage [5]

As the major burn patient suddenly faces pain, loss of control, fear and the demands of burn rehabilitation, psychiatric disorders begin to arise such as depression, anxiety and losing the will of living. And with time and neglecting dealing with these disorders, wound healing and recovery are negatively affected in comparison to normal population and even to trauma population [6]

Aim of the study is assessment of psychiatric status of burn patients during hospitalization, determination of psycological intervention methods and observation of the impact of psycological rehabilitation on healing of burn patients.

\section{Patients and Methods}

The present non-randomized, prospective, cohort study was conducted on 40 patients with partial or full-thickness burns that attend burn unit of Plastic Surgery Department, Menoufia University Hospital between January and December 2019. Approval of the ethical committee and written informed consent from all participants will be obtained.

Studied populations were divided into two groups:

- Group A (case group): 21 patients who received psychological support in the form of psychotherapy sessions, pain control methods and social support.

- Group B (control group): 19 patients with usual burn care.

\section{Inclusion criteria:}

1- Adult patients from 18-60 years old.

2- Both genders.

3- Partial thickness burns $20-40 \%$ total body surface area.

4- Full thickness burn over 10-30\% total body surface area.
5- Significant burn to special areas like face, hand, foot, joints and genitalia.

6- Patients, who could communicate verbally.

7- Patients, who had significant and obvious disfigurement that is likely to hamper social adjustment.

8- Patients, who had showed a definite need for counseling in the initial intake sessions.

\section{Exclusion criteria:}

1- Patients with major burn above $40 \%$ total body surface area or minor burn below $10 \%$ total body surface area.

2- Children and elderly.

3- Patients with chronic medical disease e.g. diabetes mellitus, hepatic disease and heart failure.

4- Burn patients with previously known psychiatric conditions like depression or anxiety.

5- Significant associated injuries like poly trauma patient or fractures.

All the cases admitted to the burn unit from two groups received the usual burn care equally in the form of: Fluid resuscitation by parkland formula according to percentage of affected total body surface area and body weight in the first 48 hours. Analgesics. Scrubbing under general anasethia in the first 24 hours. Escharotomy and fasciotomy when needed. Topical antimicrobial agent. Covering with non-adherent dressing. Early excision of eschars and deep dermal burns followed by grafting in the first 72 hours. Nutritional support. Systemic antibiotics according to culture and sensitivity. Close monitoring of patients' vitals e.g. pulse, blood pressure, respiratory rate, temperatue and glucose blood level. Continous follow up of patient's health status by full laboratory tests aspiration and burn's status by daily dressing. Maintenance fluid replacement: Both groups will be examined psychologically by Beck depression inventory scoring scale and Zung self-rating anxiety scale two times the first one at the first 48 hours and the second one before discharge.

Furthermore, case group patients will have psychological support in the form of psychotherapy sessions, pain control methods and social support.

Potent opioid drugs are the cornerstone in the management of acute pain due to burn injuries. It is suggested to initiate management with potent opioid drugs and then taper down, instead of scaling up in potency. Every service in which these drugs 
are used routinely must have access to oxygen sources, devices, and trained staff for Cardiopulmonary Cerebral (CPCR) resuscitation, and naloxone. In addition, side effects from opioid administration must be anticipated and treated aggressively. Extremely painful procedures or procedures that cannot be tolerated by the patient must be performed under general anaesthesia.

We gave all patients a scheduled Around-TheClock (ATC) prescription of paracetamol 500mg p.o. every 6 hours in adults, unless contraindicated. Adding an ATC short-cycle (maximum 3 days) prescription of Nonsteoridal Anti-Inflammatory Agents (NSAIDs) may be considered. (Ibuprofen 400mg Per Os (PO) every 8 hoursor diclofenac $50 \mathrm{mg}$ PO every 12 hours, $75 \mathrm{mg}$ IV every 12 hours in adults), and ATC paracetamol in noncritically ill patients with preserved oral route during the acute burn phase.

A weak opioid agonist should be added ATC to the pharmacological regimen, including tramadol 50 to $100 \mathrm{mg}$ Intravenous (IV) every 6 to 8 hours maximum $400 \mathrm{mg} /$ day in adults, or codeine 30 to $60 \mathrm{mg}$ PO every 6 hours in adults, maximum 240 $\mathrm{mg} /$ day.

If these measures are not sufficient to achieve adequate pain control, pharmacological management with a scheduled strong opioid agonist should be initiated, making sure that the weak opioid administration is discontinued first, as follows: Low-dose intravenous morphine titration (1-2mg in adults) every 5 to 10 minutes until pain control is achieved or until the patient is drowsy, titrating the whole dose every 4 to 6 hours if persistence of the cause of pain is anticipated.

If no strong opioid is available, ATC administration of an agonist-antagonist opioid-like nalbuphine $0.4 \mathrm{mg} / \mathrm{kg} /$ dose every 4 to 6 hours may be prescribed instead of the weak opioid.

There are several options with high-quality evidence for the management of pain caused by procedures in burn patients such as dressings, debridements and grafting. The first is the application of a strong opioid before the procedure (morphine $0.05-0.2 \mathrm{mg} / \mathrm{kg} 10$ minutes before, or fentanyl $1 \mathrm{~g} / \mathrm{kg} 5$ minutes before) or the use of PCA associated with a benzodiazepine before the procedure (midazolam $0.05 \mathrm{mg} / \mathrm{kg} 30$ minutes-1 hour before). The association with ketamine has been used widely for more than 40 years in burn patients, but its use has diminished because of the side effects, which can be avoided when used together with a benzodiazepine. Ketamine at a dose of $1 \mathrm{mg} / \mathrm{kg}$ associated with a benzodiazepine (midazolam $0.05 \mathrm{mg} / \mathrm{kg}$ ) and an antisialagogue (atropine $0.01 \mathrm{mg} / \mathrm{kg}$ ) is an excellent analgesic option, provided it is administered in centers where the anesthetist is present.

Another combination of drugs with excellent analgesic effect for procedure-associated pain is dexmedetomidine in a $1 \mathrm{~g} / \mathrm{k}$ bolus, associated with a bolus dose of ketamine $2 \mathrm{mg} / \mathrm{kg}$ and tramadol $1 \mathrm{mg} / \mathrm{kg}$, in intermediate care facilities, with presence of staff trained in CPCR or of the anaesthetist.

The plastic surgery anesthesia service should think about using preventive systemic analgesia with NSAIDs or systemic paracetamol before the procedure, and tumescent anaesthesia, regional blocks, or topical lidocaine for dressing changes in an attempt at providing residual analgesic effect after the surgical-anesthetic procedure. In stable patients who tolerate well the oral route, the use of weak opioids can be considered, including codeine at a dose of 30 to $60 \mathrm{mg}$ every 6 hours and a maximum dose of $240 \mathrm{mg} /$ day, maintaining active vigilance on side effects in order to treat them promptly. The use of conventional oxycodone at a dose of 5 to $10 \mathrm{mg}$ every 8 hours or with an extended release formula every 12 to 24 hours, methadone 2.5 to $5 \mathrm{mg}$ every 6 hours, or hydrocodone 5 to $10 \mathrm{mg}$ every 4 to 6 hours may also be considered. It is important to try not to use oral opioids for more than 10 days in order to avoid physical dependence.

Psychotherapy sessions based on Cognitive Behavioral Therapy (CBT) which is a talking therapy that can help patient manage his problems by changing the way he think and behave. It's most commonly used to treat anxiety and depression, but can be useful for other mental and physical health problems. CBT is based on the concept that patient's thoughts, feelings, physical sensations and actions are interconnected, and that negative thoughts and feelings can trap him in a vicious cycle. CBT aims to help him deal with overwhelming problems in a more positive way by breaking them down into smaller parts. Patient is shown how to change these negative patterns to improve the way he feels. Unlike some other talking treatments, CBT deals with his current problems, rather than focusing on issues from his past.

It looks for practical ways to improve patient's state of mind on a daily basis. Patients usually had two sessions a week, with each session lasting 15 to 30 minutes.

Intake interview: The contact with the patients under study began with an intake after the patient 
was admitted. The intake interview gathered the basic identification data of each patient such as age, sex, education level, income, type and cause of burns, first aid offered etc. It also collected some information on family background, adjustment problems, if any; traits, relationships with significant people etc. The details of these were collected during consecutive sessions.

Social support assessment: Emotional support through reassurance, empathy etc. Informational support means where one can get various types of support about burn injuries.

It was seen that family was the basic support group for all cases for all types of supports. However, informational support regarding management of burns was provided by hospital staff. There was a significant lack of any formal organization providing any kind of support.

\section{Patient strength assessment:}

Assessment of patient strength was done on a continuous basis. It included the following parameters. Despite the disfigurement whether the patient can do:

1- To share/open up.

2- To identify positive/supportive people/places resources that can be helpful in adjustments due to burn injury.

3- To accept the problem areas arising due to burn injury. Has the will to adapt the changes in life style/functional status etc. due to burn injury.

4- To make decisions about changes/adaptations.

It was seen that women as a group were more open than men as a group. However, it may be assigned to the fact that the counselor is a female and thus may have gender bias.

Crying and talking out were most commonly used ventilating strategies despite the gender differences.

Social aspects of disfigurement that bothered majority of the patients were:

1- A negative/low self image due to physical disfigurement basically because people will point out fingers to 'odd man out'.

2- Anxiety about 'how to manage' in future the assigned roles one is expected to play in life.

The follow-up interviews after discharge from the hospital revealed that patients had a tendency to minimize/avoid social contacts after returning home. This was due to mainly:
1- Physical pain/functional limitations in movements.

2- Hesitance felt by majority assuming people may ask questions regarding the injuriy.

3- Family members being very protective towards the patient and doing almost all possible things on their behalf.

4- Initially lack of activities that are not physically tiring but provide socialization and engagement and later on getting used to security of the home bound situation.

5- Lack of self help group with whom they can share the problems and ways of handling them.

6- Anxiety regarding family members taking on added burden of their daily life and not waiting to increase the burden.

Based on the outcomes of the above-mentioned assessments individual intervention plan was prepared for each patient. The basic interventions offered were: Counseling-emotion focused. Counseling-problem focused.

The interventions were aimed at counseling for emotion release that would help minimize stress prevent progression of negative feelings and promote positive coping strategies. This was achieved by building a good rapport with the patient, by encouraging ventilation of feelings and focusing on the residual physical and psychological abilities. Most of the time counseling for emotion release and problem solving went hand in hand.

The counseling for problem solving aimed at positive approach. The counselor and the patient and sometimes the family member jointly listed out anticipated future emotions/problems that are likely to be encountered and discussed appropriate responses to them. The patients were also encouraged to think as how they can make conscious efforts to initiate desirable and positive social responses by initiating eye contact or conversation for breaking the ice. The possible ways to satisfy the curiosity of people without hurting one's own self were discussed where ever felt necessary.

A number of other areas of concern that emerged from these sessions are listed below:

1- Meeting similar patients: The concept of self help group that can offer sharing of experiences as well as support during hospitalization and after discharge.

2- Counseling of family members in some cases. 
The continued sessions in most of the cases revealed that the adjustment after burn injury was satisfactory in most of the cases.

It was also interesting to observe that counseling for other family members was felt as garments etc. were identified as some important issues by the family members, they showed interest in ventilating feelings and working out solutions by discussing these matters with the doctor.

\section{Results}

Statistical methods: Data were collected, tabulated, statistically analyzed using an IBM personal computer with Statistical Package of Social Science (SPSS) version 20 and Epi Info 2000 programs, where the following statistics were applied.

\section{Two types of statistical analysis were done:}

Descriptive statistics: In which quantitative data were presented in the form of mean (X), Standard Deviation (SD), range, and qualitative data were presented in the form numbers (No) and percentages (\%).

I- Mean value (X).

II- Standard Deviation (SD).

Analytical statistics: Student's $t$-test $(t)$, Anova test (f), Chi-squared test $\left(\chi^{2}\right)$, Fisher's Exact Test (FET), Mann Whitney test (U), Kruskal-Wallis test $(\mathrm{KW})$ and Pearson correlation $(r)$.

The present non-randomized, prospective, cohort study was conducted on injured patients with partial or full-thickness burns that attend Burn Unit of Plastic Surgery Department, Menoufia University Hospital between January and December 2019.

Table (1): The demographic characteristics of the included patients.

\begin{tabular}{|c|c|c|}
\hline \multirow{2}{*}{$\begin{array}{l}\text { Patients' } \\
\text { characteristics }\end{array}$} & \multicolumn{2}{|c|}{$(\mathrm{n}=40)$} \\
\hline & No. & $\%$ \\
\hline $\begin{array}{l}\text { Age (years): } \\
\text { Mean } \pm \text { SD } \\
\text { Range }\end{array}$ & \multicolumn{2}{|c|}{$\begin{array}{c}31.35 \pm 10.90 \\
18-59\end{array}$} \\
\hline $\begin{array}{l}\text { Sex: } \\
\quad \text { Male } \\
\text { Female }\end{array}$ & $\begin{array}{l}29 \\
11\end{array}$ & $\begin{array}{l}72.5 \\
27.5\end{array}$ \\
\hline
\end{tabular}

*: Data are presented as mean $\pm \mathrm{SD}$, median (range), or number $(\%)$.

Table (1) shows the demographic characteristics of the included patients. The mean age of the included patients was $31.35 \pm 10.9$ years old and the majority of patients were males $(72.5 \%)$.
Table (2): The burn characteristics of the included patients.

\begin{tabular}{llc}
\hline & \multicolumn{2}{c}{$(\mathrm{n}=40)$} \\
\cline { 2 - 3 } & No. & $\%$ \\
\hline Etiology: & \multicolumn{2}{c}{45} \\
Flamed & 21 & 55 \\
Scaled & \multicolumn{2}{c}{$\mid$} \\
Burn surface area $\%:$ & \multicolumn{2}{c}{$18.45 \pm 8.51$} \\
Mean \pm SD & \multicolumn{2}{c}{$10-40$} \\
Range & \multicolumn{2}{c}{27.5} \\
Burn degree: & 11 & 15.0 \\
2nd & 6 & 57.5 \\
3rd & 23 & \\
Mixed &
\end{tabular}

*: Data are presented as mean $\pm \mathrm{SD}$, median (range), or number (\%).

Table (2) shows the burn characteristics of the included patients. Most of the patients had scaled burn $(55 \%)$ and the mean burn surface area was $18.45 \pm 8.51 \%$. The majority of the patients had mixed burn degree $(57.5 \%)$.

Table (3): The follow-up results of the included patients.

\begin{tabular}{lll}
\hline & \multicolumn{2}{c}{$(\mathrm{n}=40)$} \\
\cline { 2 - 3 } & No. & $\%$ \\
\hline Time to heal in days: & \multicolumn{2}{c}{$11.5 \pm 4.5$} \\
Mean \pm SD & \multicolumn{2}{c}{$5-20$} \\
Range & \multicolumn{2}{c}{} \\
Satisfaction: & 32 & 80 \\
Satisfied & 8 & 20 \\
Not & 8 \\
\hline
\end{tabular}

*: Data are presented as mean $\pm \mathrm{SD}$, median (range), or number (\%).

Table (3) shows that the follow-up results of the included patients. The mean time to heal was $11.5 \pm 4.5$ days and $80 \%$ of the patients were satisfied.

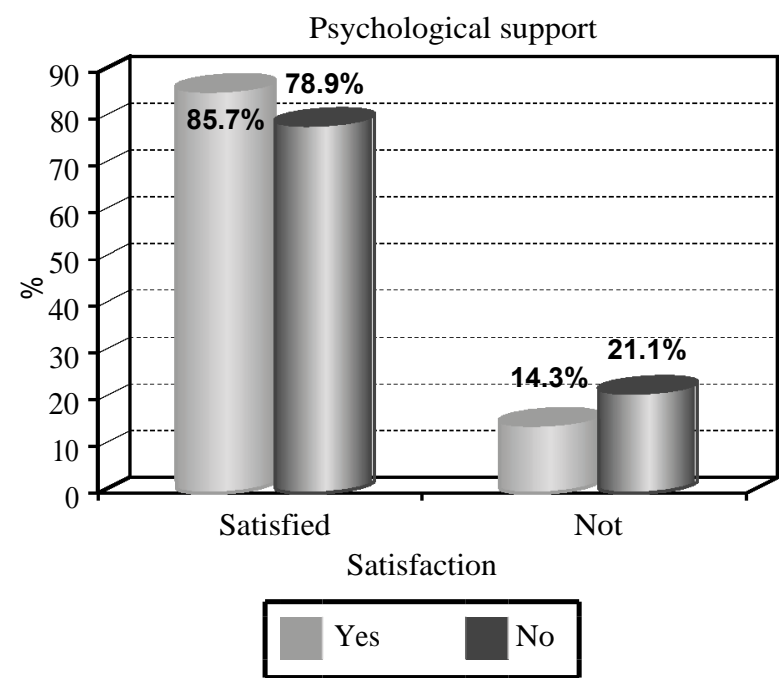

Chart (1): The follow-up results of the included patients. 
Table (4): The complications of the included patients.

\begin{tabular}{lll}
\hline & \multicolumn{2}{c}{$(\mathrm{n}=40)$} \\
\cline { 2 - 3 } & No. & $\%$ \\
\hline Early complications: & 22 & 55 \\
No & 12 & 30 \\
Infection & 6 & 15 \\
Pigmentation & & \\
Late complications: & 32 & 80 \\
No & 2 & 5 \\
Hypertrophic scar & 2 & 5 \\
Keloid & 4 & 10 \\
Pigmentation & & \\
\hline
\end{tabular}

Table (4) shows the complications of the included patients. $30 \%$ of the patients had early infection and $15 \%$ had early pigmentation. In addition, $10 \%$ of the patients had late pigmentation and only $5 \%$ had infection and hypertrophic scars, each.

Table (5): The baseline BDI score of the included patients.

\begin{tabular}{lcc}
\hline & \multicolumn{2}{c}{$(\mathrm{n}=40)$} \\
\cline { 2 - 3 } & No. & $\%$ \\
\hline BDI: & \multicolumn{2}{c}{$27.7 \pm 16.2$} \\
Mean \pm SD & \multicolumn{2}{c}{8.50} \\
Range & \multicolumn{2}{c}{5} \\
BDI Category: & 2 & 35 \\
Minimal depression & 14 & 40 \\
Mild depression & 16 & 20 \\
Moderate depression & 8 & \\
Severe depression & \multicolumn{2}{c}{}
\end{tabular}

Table (5) shows that the baseline BDI score of the included patients. The mean BDI was 27.7 $\pm 16.2 ; 40 \%$ of the patients had moderate depression and $20 \%$ had severe depression.

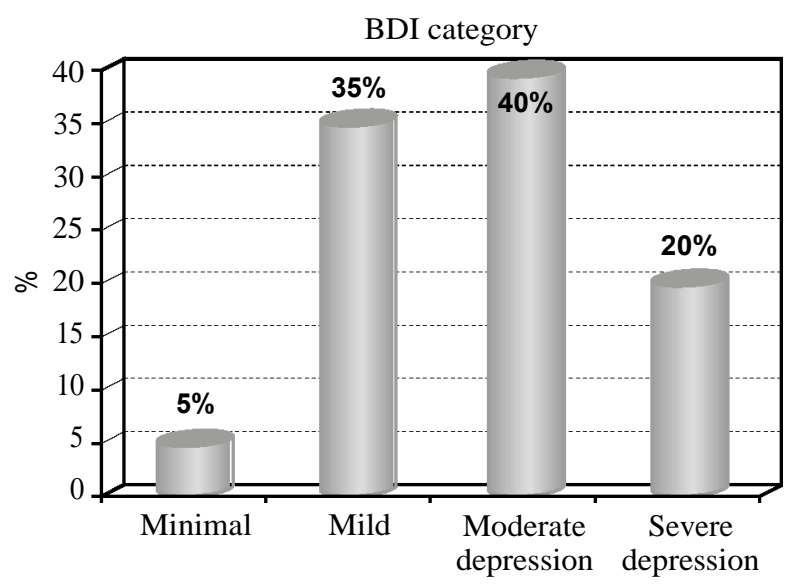

Chart (2): The baseline BDI score of the included patients.

\section{Discussion}

Treatment of people with burn injuries includes recovery of optimal function for survivors to fully participate in society, psychologically and physically. Increased likelihood of physical survival has led to greater concern for potential psychological morbidity for the burn survivor [7]

Burn injuries can be one of the most traumatic experiences of a person's life. Research has documented a wide range of effects that a burn injury can have on people who sustain the injury and their family, including depression, anxiety, social difficulties, and appearance-related concerns. However, while it is acknowledged that these issues may be far-reaching and long-lasting, little research has explored interventions to facilitate healthy psychosocial adjustment after a burn injury [8] .

The impact of psychological factors on wound healing is an important and interesting field of research. There is sound evidence to demonstrate that the effects of psychological stress on wound healing are significant. The number of recently published reviews that focus on psychological influences on wound healing, post-surgical recovery and psychological interventions that have impacted on healing is in growing interest [6].

Despite the growing interest in the application of psychological interventions for burn affected patients, there is a scarcity in the published literature which assess the utility of these interventions. Thus, we conducted the present study in order to evaluate the psychiatric status of burn patients during hospitalization, determination of psychological intervention methods and observation of impact of psychological rehabilitation on healing of burn patients.

The present non-randomized, prospective, cohort study was conducted on injured patients with partial or full-thickness burns that attend Burn Unit of Plastic Surgery Department, Menoufia University Hospital between January and December 2019.

Studied populations were divided into two groups: Group A (case group): 21 patients who received psychological support in the form of supportive psychotherapy sessions, pain control methods and social support. Group B (control group): 19 patients with usual burn care.

Burn injuries can vary from relatively minor insults to severe debilitating injuries. Over 50\% of burn injuries involve the head and neck region 
and can be caused by flame, electrical current, steam, hot substances, and chemicals [9]

In the present study, most of the patients had scaled burn $(55 \%)$ and the mean burn surface area was $18.45 \pm 8.51 \%$. The majority of the patients had mixed burn degree $(57.5 \%)$.

In line with our findings, it was conducted in a retrospective study on 350 patients with facial burns treated in 2006-2011 period in Department of Plastic and Reconstructive Surgery Kosova. Scalds were the most common cause of burns $(44.6 \%)$, followed by fire $(31.1 \%)$. The majority of the patients had mixed burns [10]

A Chinese study described the hospitalized population with burn injuries in eight burn centers in China between 2011 and 2015. This 5-year retrospective review included all patients with burns in a database at eight institutions. A total of 1126 patients were included: $803(71.3 \%)$ male patients and $323(28.7 \%)$ female patients. Scalds were the most common cause of burns ( 476 , $42.27 \%)$, followed by fire $(457,40.59 \%)$. The majority of the patients had mixed burns [11].

In another Chinese study which investigated the epidemiology and outcomes of burn patients in a major burn center in southwest China between 2011 and 2015 to provide guidance for burn prevention. Of the 6,325 included burn patients, $66.8 \%$ were male and $34.7 \%$ were $0 \sim 6$ years old. The incidence of burns peaked in autumn. Scald was the most common cause of burns. The majority of the patients had mixed burns [12]

So far, the Beck Depression Inventory-II (BDIII) has become one of the most widely used measures to assess depressive symptoms and their severity in adolescents and adults. The BDI-II is a 21-item self-report measure that taps major depression symptoms according to diagnostic criteria listed in the Diagnostic and Statistical Manual for Mental Disorders. Items are summed to create a total score, with higher scores indicating higher levels of depression. It is worth noting that the BDI-II is not only extensively applied for research purposes but also in clinical practice, being the third test most used among Spanish professionals [13].

In the present study, we used BDI-II to assess depressive symptoms among patients affected by burn. Before the psychological interventions, we found that the mean BDI was $27.7 \pm 16.2 ; 40 \%$ of the patients had moderate depression and $20 \%$ had severe depression.
In concordance with our findings, a recent study assessed level of anxiety, depression and selfesteem in burn patients. This cross-sectional study included 100 patients with burn injuries admitted to a tertiary care private hospital in an urban metropolis in India. Almost 32\% of the patients had moderate depression [14]

Likewise, a prospective-observational study in a University-affiliated hospital on 50 burn patients participated in the study. The prevalence of moderate depression was $36.9 \%$; and extreme depression was $42.1 \%$ [15]

The socio-demographic distribution of patients developing Anxiety and Depression with the effects of burn related factors on development of Anxiety and Depression. A case series was conducted at the Department of Burn, Pakistan Ordinance Factory Hospital, Wah Cantt. Fifty patients were included in the study. Depression was seen amongst $29(58 \%)$ patients. Thirteen $(26 \%)$ patients had mild, 7 (14\%) had moderate and $9(18 \%)$ had severe depressive symptoms [16]

With regard to BDI-II score after the intervention, we found that there was statistically significant difference between studied groups in terms of BID score at the end of follow-up ( $p=0.0045)$, with higher score in patients with no psychological support. On the other hand, there was no statistically significant difference between studied groups in terms of rate of BDI category ( $p=0.32$ ).

It was also noted that study group patients improved clinically with psychological rehabilitation in many ways such as patient's compliance for treatment and wound care, significant decrease in the dosage of analgesics needed by the patient and shorter hospital stay period.

To the best of our knowledge, our study is the first trial of its kind that assessed the role of psychological intervention on depression during hospitalization of burn patients.

The Zung Self-Rating Anxiety Scale (ZSRAS) is a commonly utilized norm-referenced scale. The ZSRAS is a 20-item Likert scale covering symptoms that were identified in factor analytic studies of the syndrome of anxiety. Items tap psychological and physiological symptoms and are rated by respondents according to how each applied to them within the past week, using a 4-point scale ranging from 1 (none, or a little of the time) to 4 (most, or all of the time). The scale has a raw score range of 20 to 80 points. The raw score is then converted to an index score by dividing the raw score by the 
maximum score (80) and either expressing this as a decimal or multiplying by 100 to express it as a whole number with an index score range of 25 to 100 . Index scores of 25 to 49 indicate nil anxiety, 50-59 indicate mild to moderate anxiety, 60-69 indicate moderate to severe anxiety, and scores over 70 indicate severe anxiety [17].

In the present study, in the pre-intervention period, we found that the mean SDS was $47.7 \pm 18.4$; $35 \%$ of the patients had mild-to-moderate anxiety.

However, other reports showed significantly much higher rate of anxiety than our report. The study conducted [16] on burn patients found that following the burn $82 \%$ of the patients have anxiety.

Another study [14] reported that $49 \%$ of the burn patients had moderate-to-severe anxiety.

Assessment of the frequency of anxiety and depression in burn victims with assessment the socio-demographic distribution of such cases. 100 cases were selected among all the patients admitted to burns ward of McGann Hospital Shimoga Institute of Medical Sciences, during the two-year period from January 2011 to December 2012, with $10 \%$ to $50 \%$ burns. Anxiety was seen amongst $78 \%$ of cases, out of which $30 \%$ had mild, $32 \%$ had moderate and remaining $38 \%$ had severe anxiety scores [18].

After the intervention, we found that there was statistically significant difference between studied groups in terms of ZSRAS score at the end of follow-up ( $p=0.0045)$, with higher score in patients with no psychological support. On the other hand, there was no statistically significant difference between studied groups in terms of rate of ZSRAS category $(p=0.32)$.

Although we found no published adults' study about the impact of psychological intervention on anxiety during hospitalization of burn patients. Previous reports assessed the impact of those interventions in pediatric patients. Another study conducted as prospective, randomized controlled trial was conducted, comparing psychological intervention versus standard care in relation to pain and anxiety scores of children undergoing their initial burn dressing change. 50 subjects were recruited in each treatment group. Combined and scaled pain and anxiety scores in the psychological intervention group were significantly less than in the standard treatment group $(p=0.03)$ [19] .

A number of studies have demonstrated a link between psychological distress and longer hospital stays following a burn injury, where longer stays are taken to indicate longer wound healing time [20].

In our study, we found that there was significant difference between studied groups in terms of hospital stay $(p=0.001)$, with shorter hospital stays among patients receiving psychological support.

Again, to the best of our knowledge, our study is the first trial of its kind that assessed the role of psychological intervention on hospital stay in burn patients.

\section{Conclusion:}

Psychological interventions appear to effectively reduce burn patient psychological morbidities. We demonstrated that psychological interventions significantly reduce the risk of depression, anxiety, and hospital stay compared to usual care. These findings are very important as it confirms the promising role of psychological interventions in burn patients. Nevertheless, further studies with rigorous design, large sample size and multiregional cooperation are required.

\section{References}

1- McCLURE J. and MOORE E.C.: Burns and inhalational injury. Anasethia and intensive care medicine, 12: 9, 2011.

2- KLEIN B.M.: Thermal, chemical and electrical injuries. Grabb and Smith's plastic surgery, sixth edition, 2007.

3- WISELY J.: The impact of psychological distress on the healing of burns. Wounds UK, 9 (3): 14-7, 2013.

4- TOBIASEN J.M. and HIEBERT J.M.: Burns and adjustment to injury: Do psychological coping strategies help? J. Trauma, 25 (12): 1151-5, 1985.

5- WIECHMAN S.A. and PATTERSON D.R.: Psychosocial aspects of burn injuries. BMJ, 329 (7462): 391-3, 2004.

6- HASSAN M.S. and SOLTAN M.R.: The Impact of Remodeling Psychological Status on Prognosis of Major Burns Patients. Egypt, J. Plast. Reconstr. Surg., 44 (2): 311-7, 2020

7- HEATH J., WILLIAMSON H., WILLIAMS L. and HARCOURT D.: Parent-perceived isolation and barriers to psychosocial support: A qualitative study to investigate how peer support might help parents of burn-injured children. Scars, burns \& healing, 4, 2059513118763801 , 2018.

8- WILLIAMS H.M., HUNTER K., CLAPHAM K., RYDER C., KIMBLE R., et al.: Efficacy and cultural appropriateness of psychosocial interventions for paediatric burn patients and caregivers: A systematic review. BMC Public Health; 20, 2020.

9- KARA Y.A.: Burn Etiology and Pathogenesis. Hot Top. Burn Inj, 2018. 
10- ZATRIQI V., ARIFI H., ZATRIQI S., DUCI S., RRECAJ S., et al.: Facial Burns-Our Experience. Mater Socio Medica, 25: 26, 2013.

11- TIAN H., WANG L., XIE W., SHEN C., GUO G., et al.: Epidemiologic and clinical characteristics of severe burn patients: Results of a retrospective multicenter study in China, 2011-2015. Burn Trauma, 6, 2018.

12- LI H., YAO Z., TAN J., ZHOU J., LI Y., et al.: Epidemiology and outcome analysis of 6325 burn patients: A fiveyear retrospective study in a major burn center in Southwest China. Sci. Rep., 7: 46066, 2017.

13- GARCÍA-BATISTA Z.E., GUERRA-PEÑA K., CANOVINDEL A., HERRERA-MARTÍNEZ S.X. and MEDRANO L.A.: Validity and reliability of the beck depression inventory (BDI-II) in general and hospital population of Dominican Republic. PLoS One, 13, 2018.

14- JAIN M., KHADILKAR N. and De SOUSA A.: Burnrelated factors affecting anxiety, depression and selfesteem in burn patients: An exploratory study. Ann. Burns Fire Disasters, 30: 30-4, 2017.

15-PAVONI V., GIANESELLO L., PAPARELLA L., BUONINSEGNI L.T. and BARBONI E.: Outcome predictors and quality of life of severe burn patients admitted to intensive care unit. Scand J. Trauma Resusc. Emerg. Med., $18,2010$.

16- TABASSUM A., FATIMA A. and MA N.M.: Anxiety and depression in burn patients. J. Ayub. Med. Coll Abbottabad., 21: 137-41, 2009.

17- DUNSTAN D.A. and SCOTT N.: Assigning Clinical Significance and Symptom Severity Using the Zung Scales: Levels of Misclassification Arising from Confusion between Index and Raw Scores. Depress. Res. Treat., 2018.

18- ARIF M. and RAMPRASAD K. S.: Prevalence of anxiety and depression in burns patients in a tertiary care hospital. J. Dental. Med. Sci. (IOSR-JDMS), 10 (4): 6-9, 2013.

19- HYLAND E.J., D'CRUZ R., HARVEY J.G., MOIR J., PARKINSON C. and HOLLAND A.J.A.: An assessment of early Child Life Therapy pain and anxiety management: A prospective randomised controlled trial. Burns, 41: 1642-52, 2015.

20- JULIE W.: The impact of psychological distress on the healing of burns. Wounds UK, 9: 14-7, 2013.
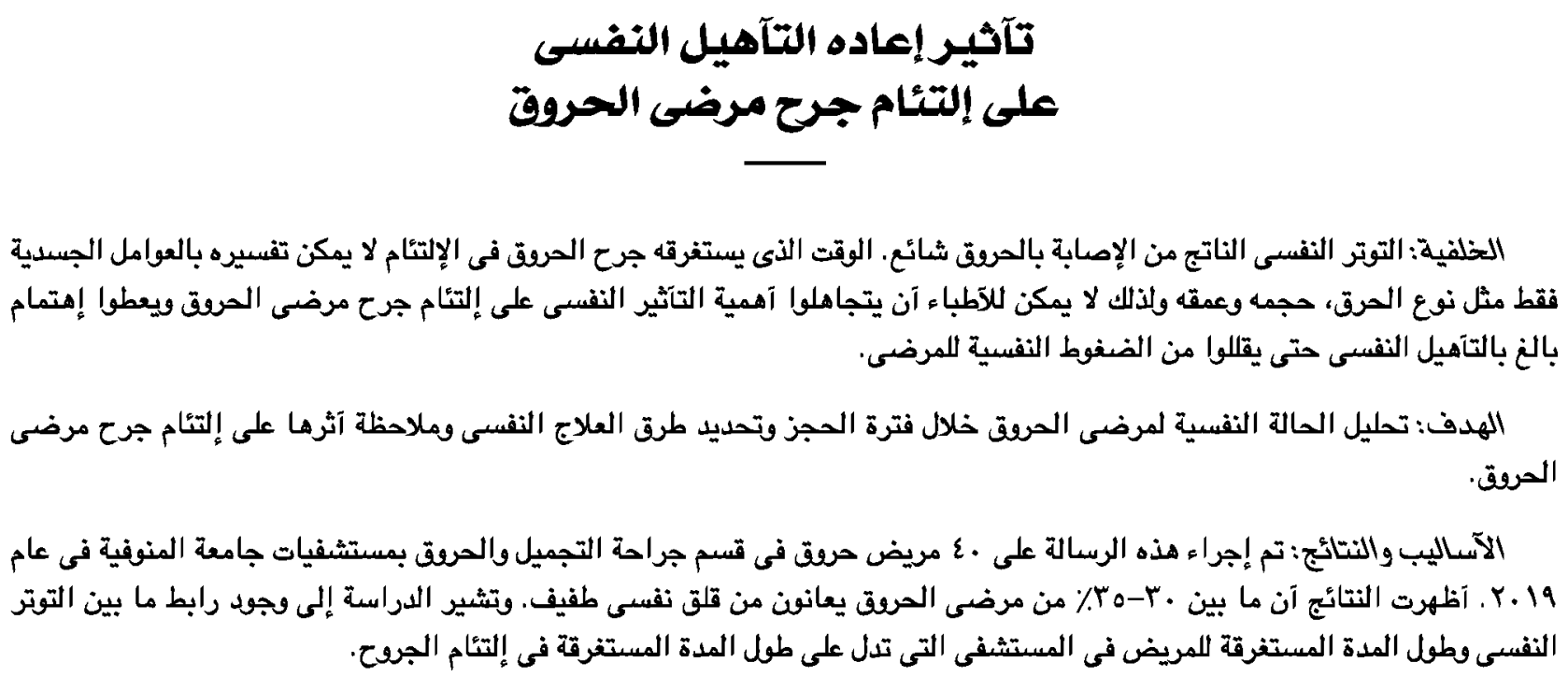

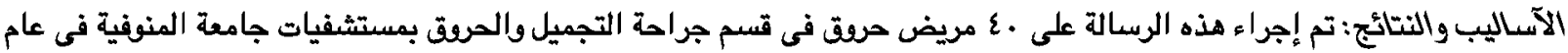

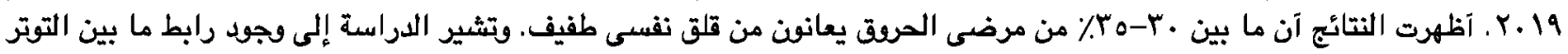

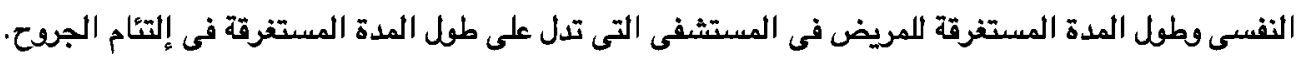

الخلاصة: وسيائل العلاج النفسى لمرضى الحرق تقلل الإضطرابات النفسية بوضوح وتحد من الإكتئاب والقلق النفسى وهذه النتائج تؤكد 